\title{
Future Perspectives of Health Care: Closing Remarks
}

\author{
Gørill Haugan and Monica Eriksson
}

\section{Abstract}

The Covid-19 pandemic has demonstrated the vulnerability of our health care systems as well as our societies. During the year of 2020, we have witnessed how whole societies globally have been in a turbulent state of transformation finding strategies to manage the difficulties caused by the pandemic. At first glance, the health promotion perspective might seem far away from handling the serious impacts caused by the Covid-19 pandemic. However, as health promotion is about enabling people to increase control over their health and its determinants, paradoxically health promotion seems to be ever more important in times of crisis and pandemics. Probably, in the future, pandemics will be a part of the global picture along with the noncommunicable diseases. These facts strongly demand the health care services to reorient in a health promoting direction.

The IUHPE Global Working Group on Salutogenesis suggests that health promotion competencies along with a reorientation of professional leadership towards salutogenesis, empowerment and participation are required. More specifically, the IUHPE Group recommends that the overall salutogenic model of health and the concept of SOC should be further advanced and applied beyond the health sector, followed by the design of salutogenic interventions and change processes in complex systems.

\section{Keywords}

Health promotion $\cdot$ Pandemics $\cdot$ Noncommunicable diseases $\cdot$ Reorienting the health-care services $\cdot$ Salutogenesis $\cdot$ Salutary factors

\section{G. Haugan $(\bowtie)$ \\ Department of Public Health and Nursing, NTNU Norwegian University of Science and Technology, Trondheim, Norway}

Faculty of Nursing and Health Science,

Nord University, Levanger, Norway

e-mail: gorill.haugan@ntnu.no,

gorill.haugan@nord.no

\section{Eriksson}

Department of Health Sciences, University West, Trollhattan, Sweden

e-mail: monica.eriksson@hv.se

\subsection{Future Perspective}

The present condition gives us directions in which to look forward; the year of 2020 is the year of the Covid-19 pandemic. Lately, entire cities, regions and countries have been sealed off, travelling has been banned, universities have been closed, along with shops, restaurants etc. all over the world. We have witnessed that economic, cultural and social 
activities have come to a stop, resulting in big challenges to a great number of people around the globe. Thus, health concerns have become the prevailing concern that takes precedence over all other issues. In the years to come, the health services globally will need to collaborate on handling pandemics such as the Covid-19 pandemic ruling the world during the writing process of this book. Facing the serious impacts caused by the Covid-19 pandemic, at first sight, this pandemic and the world's response to it might seem far away from the health promotion perspectives. However, in a recently published Editorial in Health Promotion International, Van den Broucke [1] (p. 181) highlights the important role of health promotion in the time of crisis and pandemics by saying “...Enabling people to increase control over their health and its determinants is at the core of health promotion. As such, health promotion may paradoxically be more important in this time of crisis than ever before". Probably, in the future pandemics will be a part of the global picture along with the so-called non-communicable diseases (NCD) which covers chronic illnesses such as cancer, dementia, heart failure, diabetes as well as mental health issues. In this book, we have highlighted the need of health promotion as an integrated aspect of the treatment and care of patients with various NCDs. NCD Countdown 2030 [2] is an independent collaboration to inform policies that aim to reduce the worldwide burden of NCDs, and to ensure accountability towards this aim. In 2016, an estimated 40.5 million (71\%) of the 56.9 million worldwide deaths were from NCDs. Of these, an estimated 1.7 million (4\% of NCD deaths) occurred in people younger than 30 years of age, 15.2 million (38\%) in people aged between 30 years and 70 years, and 23.6 million $(58 \%)$ in people aged 70 years and older. An estimated 32.2 million NCD deaths $(80 \%)$ were due to cancers, cardiovascular diseases, chronic respiratory diseases, and diabetes, and another 8.3 million (20\%) were from other NCDs [2]. These facts strongly demand the health care services to reorient in a health promoting direction. As Van den Broucke [1] argued in his editorial, the pandemic has shown how vulnerable health care services may be, and not only the health care sector; whole societies are in a turbulent state of transformation in need of ways and strategies to manage.

To return to the health care sector, the IUHPE Global Working Group on Salutogenesis states in a position article that one way is to position health promotion competencies as an essential framework to reorient health care services [3] (p. 9). In addition, the professional leadership must be reoriented towards salutogenesis, empowerment and participation. To move forward with the concept of salutogenesis as a sound scientific base for health promotion, some suggestions have been given:

1. To advance the overall salutogenic model of health

2. To advance the concept of SOC

3. To define and design salutogenic interventions and change processes in complex systems

4. To apply salutogenesis beyond the health sector [3]

\subsubsection{Advancing the Overall Salutogenic Model of Health}

The IUHPE Group states that the salutogenic model of health needs an additional positive health continuum and a path of positive health development linking resources to this new continuum [3]. During the last decades, a broader literature on the ease-end of the health continuum has emerged emphasizing among others the importance of developing individuals' personal potential and functioning, supporting people's perception of self-fulfillment, purpose and meaning-in-life, thriving, social attractiveness and making a valuable contribution to society [4-6]. Considering these developments, the IUHPE group recommends the addition of a positive health continuum to Antonovsky's original salutogenic model [3]. Furthermore, the IUHPE Group underscores the importance of linking resources to this new continuum of positive health development; “....Resources not only immediately help people to cope better with stress (and surviving). Also, over time personal and environmental resources can help with recovery and healing... Beyond healing and recovery, resources can 
directly promote health, wellbeing and thrivingeven in the absence of current or previous adversarial life situations" [3] (p. 3). Consequently, in line with the Health Development Model [7] which proposes that pathogenesis and salutogenesis are two complementary perspectives on health development, GRRs and SRRs ${ }^{1}$ are seen to facilitate and nurture positive health; thus direct paths of positive health development have been added to the salutogenic health model [3].

\subsubsection{Advancing the Concept of SOC}

The second point underscores a need for advancing the SOC concept; many translations of the Orientation to Life Questionnaire (OLQ) and the evidence on SOC have provided confidence that the SOC construct is measurable. However, the substance, content, wording and dimensionality of the SOC construct have yet to be explored. Research on SOC utilizing other methodological approaches than Antonovsky used accompanied by a replicability of Antonovsky's qualitative analyses and findings are highly welcome [3] (p. 4). Further, there is a need of developing new questionnaires for measuring salutary factors for health and wellbeing. Some attempts can be found, for example The Salutogenic Health Indicator Scale (SHIS) [8]. In Sweden, an ongoing research study has developed and tested a new scale, adapted for nurses' work situation and to be used in health care, The Salutogenic Survey on Sustainable Working life-Nurses (SalWork-N) (www.hv.se).

\subsubsection{Salutogenic Interventions and Change Processes}

Thirdly, strengthening the SOC through health promotion intervention is key. Purposefully designed salutogenic interventions and change processes are needed; therefore, explicit salutogenic intervention theories building on and inte-

${ }^{1}$ GRR = Generalized Resistance Resources; SRR = Specific Resistance Resources, central concepts in salutogenesis. grating key elements of salutogenesis should be developed [3]. Antonovsky stated that SOC [9] is formed by three kinds of life experiences: (1) consistency (strengthening comprehensibility), (2) underload-overload balance (strengthening manageability), and (3) participation in socially valued decision making (strengthening meaningfulness). Accordingly, further knowledge about these kinds of life experiences and how they can be assessed (quantitatively and qualitatively) in different contexts as well as on different system levels are required [3] (p. 5). To reorient health care, it is important to capture factors and initiatives that nurses along with other health professionals consider health promoting resources in their everyday clinical practice. We must learn from their experiences and integrate this new knowledge in education for health professionals.

\subsubsection{Applying Salutogenesis Beyond Health Sector}

Finally, applying salutogenesis and SOC to other fields beyond the individual health issues might be valuable as we can learn from other fields for health research. For instance, intergroup relations are vital in peoples' daily life at work, in leisure, in the communities and the municipalities, etc. Therefore, we need to more fully examine the differential benefits and potential harm of SOC on the individual, group and intergroup as well as organizational and system levels [3]. In a public health perspective, such knowledge seems fruitful for the development of health promoting workplaces, health promoting hospitals, health promoting communities, municipalities, schools, kinder gardens, living areas, etc.

\subsection{Health Promotion Is About Thriving and Enabling People to Increase Control over Their Health}

The health promotion perspective as well as the salutogenic theory of health is based in the idea that every single person has a health, which varies and moves along the health continuum between 
dis-ease and ease. Antonovsky [9] (p. 14) raised the question: How can we understand movement of people in the direction of the health end of the continuum?'-note, all people, wherever they are at any given time, from the terminal patient to the vigorous adolescent - we cannot be content with an answer limited to 'by being low on risk factors'. A salutogenic orientation, then, as the basis for health promotion, directs both research and action efforts to encompass all persons, wherever they are on the continuum, and to focus on salutary factors. Beyond survival, health promotion is about thriving and enabling people to increase control over their health [10]. The salutogenic model is useful for all fields of health care and therefore helpful in the reorienting of the health care services.

\subsection{A reorientation of Health Care by Implementing Salutogenesis}

We started this book showing a figure (Fig. 1.4) describing the salutogenic umbrella adjusted for health care settings. We also choose to end the book with the same figure (Fig. 26.1). Some of the umbrella concepts are described in this book, whilst some remain to be explored. However, to our knowledge this book is the first one to highlight and emphasize concepts closely related to the sense of coherence (SOC) in general, and to health care systems in particular. There is a need to continue exploring other salutogenic concepts to strengthen the salutogenic theory, but also to contribute to the development of nursing and the health sciences. Among others, concepts such as empathy, humour, learned optimism and learned hopefulness, would be interesting.

Moreover, the implementation of the concepts and the salutogenic approach to health and wellbeing in a systematic way is highly needed. An integration would benefit both staff and patients. Such an effort could begin with an education, i.e. salus education proposed in Chap. 15, continuing with a focus on learning processes to finally end with the development of a new way of working in health care settings, which means that salutary factors are recognized, identified and used in a health promoting manner. In other words, reorienting the health care services according to the

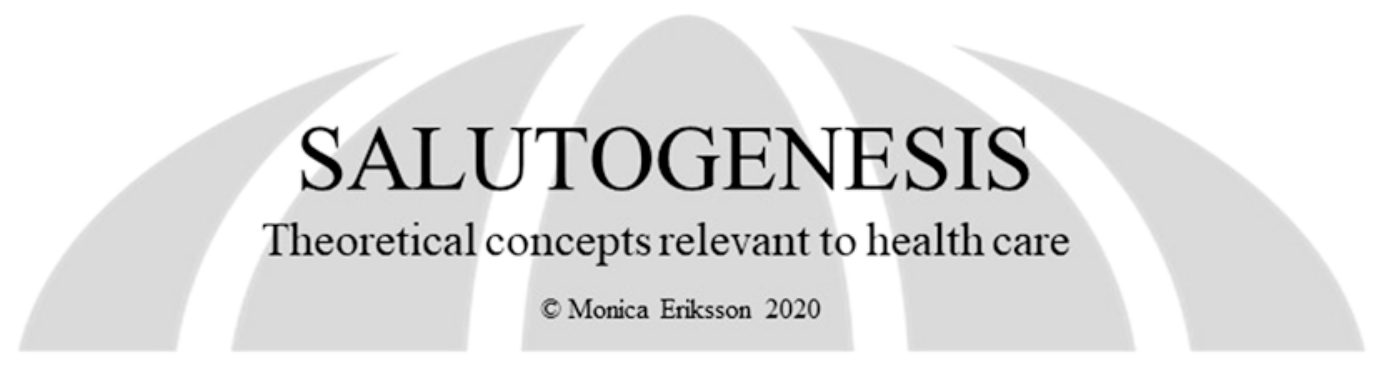

\section{Social support | Empowerment | Flourishing | Sense of Coherence | Dignity | Belonging Self-efficacy | Self-transcendence | Hope | Will to meaning | Willpower | Connectedness Salutogenic nursing | Nurse-patient interaction |Person-centered care | Inner strength Bodyknowledging | Coping}

Reasonableness | Resilience | Learned resourcefulness | Attachment | Empathy | Wellbeing Learned hopefulness | Humour | Gratitude | Quality of Life |Flow | Hardiness | Social capital Locus of Control $\mid$ Ecological system theory | Interdisciplinarity | Cultural capital | Thriving Posttraumatic Personal Growth | Learned optimism | Slow nursing

Fig. 26.1 The salutogenic umbrella: theoretical concepts relevant to health care. (Reproduced with permission from Folkhälsan Research Center, Lindstrom \& Eriksson) [4] 
Ottawa Charter for health promotion. Already in the mid-1990s Antonovsky argued the salutogenic orientation as the theoretical basis for health promotion, directing both research and action efforts particularly useful for all fields of health care [9] (p. 18). The progress of new salutogenic models of health has been limited. An attempt to fill this knowledge gap can be seen in an article on "The Synergy Model of Health", which integrates salutogenesis and the assets model in a framework of Bronfenbrenner's ecological theory of human development [11].

When reviewing research on SOC we have become aware of how limited the research on working conditions of health care personnel is. There is a lot of research by nurses and other health professionals on different patient groups including measuring the SOC. However, salutogenic research on how health professionals' health and wellbeing can be maintained and developed is scarce. The same applies to knowledge about health professionals' SOC. For instance, have nurses forgotten themselves in their quest to do good for the patients? We here argue that research must also focus on the work environment and salutary factors for developing a sustainable working life for nurses and other health professionals. However, some attempts to fill this knowledge gap can be seen. A longitudinal study on the influence of a health promoting work environment [12] as well as a study on work-related SOC and its longitudinal relationship with work engagement and job satisfaction [13] were recently conducted in Norwegian nursing homes. Correspondingly, studies focusing on the working culture in nursing homes have shown empowerment, participation and influence over the work situation to be vital aspects for care quality $[14,15]$. Moreover, SOC was newly measured among Swedish hospital nursing staff [16]. They found that on a national level, nurses reported weaker SOC than the general population, but stronger in an international comparison of nurses. Nurses found their work difficult to manage, but meaningful. Many nurses want to leave their workplaces and even the profession, as they found the work situation too stressful for their health. This is a worldwide dilemma. Another Swedish study among hospital nurses explored salutary factors for a sustainable working life [17]; having fun at work, being acknowledged, feeling togetherness in the team, having varying tasks with a manageable workload, good interaction between colleagues and patients, doing good work, feeling committed to and pride in the professional role, and having a balance between work and leisure time were found to be factors that made them stronger, which in turn explained why they stayed. Similar findings have been seen in Norway [18, 19]. Further research on salutary factors is needed.

\subsection{Closing Remarks}

In this book, we have highlighted the need to work to promote health in both hospitals and the municipality health care; treatment and care of patients with chronic diseases and the so-called non-communicable diseases (NCD) will be fundamental in the years to come. Our point of departure has been to focus attention on health promotion and salutogenesis in health care settings. We argue the salutogenic theory of health to be appropriate to guide health promotion in the health services. In a lecture at the Nordic School of Public Health (NHV) Antonovsky lectured about salutogenesis and health [20]. He called on to think salutogenically and act salutogenically. To use his own words, "the key lies in a society and in people who care about others" [20]. Nurses and the other health care professions are just such people who cares!

\section{References}

1. Van den Broucke S. Why health promotion matters to the COVID-19 pandemic, and vice versa. Health Promot Int. 2020;35:181-6.

2. NCD Countdown 2030 Collaborators. NCD Countdown 2030: worldwide trends in noncommunicable disease mortality and progress towards Sustainable Development Goal target. Lancet. 2018;22(392):1072-88.

3. Bauer GF, Roy M, Bakibinga P, Contu P, Downe S, Eriksson M, et al. Future directions for the concept of salutogenesis: a position article. Health Promot Int. 2019;2019:1-9. 
4. Lindström B, Eriksson M. The Hitchhiker's guide to salutogenesis. Salutogenic pathways to health promotion. Helsinki: Folkhälsan Research Center; 2010.

5. Commers MJ. Determinants of health: theory, understanding, portrayal, policy. Dordrecht: Springer; 2002.

6. Pelikan JM. Understanding differentiation of health in late modernity - by use of sociological system theory. In: McQueen DV, Kickbusch I, Potvin L, Pelikan JM, Balbo L, Abel T, editors. Health and modernity: the role of theory in health promotion. New York: Springer Scientific Publisher; 2007. p. 74-102.

7. Bauer GF, Davies JK, Pelikan JM. The EUHPID health development model for the classification of public health indicators. Health Promot Int. 2006;21:153-9.

8. Bringsén Å, Andersson HI, Ejlertsson G. Development and quality analysis of the Salutogenic Health Indicator Scale (SHIS). Scand J Publ Health. 2009;37(1):13-9.

9. Antonovsky A. The salutogenic model as a theory to guide health promotion. Health Promot Int. 1996;11(1):11-8.

10. WHO. The Ottawa Charter for health promotion: an international conference on health promotion. The Move towards a New Public Health. Copenhagen; 1986.

11. Pérez Wilson P, Marcos Marcos J, Morgan A, Eriksson M, Lindström B, Alvarez Dardet C. "The synergy model of health" - an integration of salutogenesis theory and health assets model. Health Promot Int. 2020; in press.

12. Grødal K, Innstrand ST, Haugan G, Andre B. Affective organizational commitment among nursing home employees: a longitudinal study on the influence of a health-promoting work environment. Nurs Open. 2019;6(4):1414-23.

13. Grødal K, Innstrand ST, Haugan G, Andre B. Workrelated sense of coherence and longitudinal relationships with work engagement and job satisfaction. Scand J Work Organ Psychol. 2019;4(1): $1-11$.

14. Andre B, Sjøvold E. What characterizes the work culture at a hospital unit that successfully implements change-a correlation study. BMC Health Serv Res. 2017; $17: 486$.

15. Andre B, Sjøvold E, Rannestad T, Ringdal GI. The impact of work culture on quality of care in nursing homes-a review study. Scand J Caring Sci. 2014;28(3):449-57.

16. Eriksson M, Kerekes N, Brink P, Pennbrant S, Nunstedt $\mathrm{H}$. The level of sense of coherence among Swedish nursing staff. J Adv Nurs. 2019;75: 2766-72.

17. Nunstedt H, Eriksson M, Ayman O, Hillström L, Truong A, Pennbrant S. Salutary factors explaining why hospital nurses remain in work and the profession. BMC Nurs. 2020; in review.

18. Hauvik S, Haugan G. The influence of rotation work on nursing home nurses' health and quality of life. Geriatr Nurs. 2016;20(2):26-35.

19. Hauvik S, Haugan G. The importance of the turn for nurses' work situation and quality of life in nursing homes. Geriatr Nurs. 2017;2017(3):5-13.

20. Antonovsky A. Some salutogenic words of wisdom to the conferees. Lecture held at the Nordic School of Public Health (NHV), 1993. Gothenburg.

Open Access This chapter is licensed under the terms of the Creative Commons Attribution 4.0 International License (http://creativecommons.org/licenses/by/4.0/), which permits use, sharing, adaptation, distribution and reproduction in any medium or format, as long as you give appropriate credit to the original author(s) and the source, provide a link to the Creative Commons license and indicate if changes were made.

The images or other third party material in this chapter are included in the chapter's Creative Commons license, unless indicated otherwise in a credit line to the material. If material is not included in the chapter's Creative Commons license and your intended use is not permitted by statutory regulation or exceeds the permitted use, you will need to obtain permission directly from the copyright holder. 